
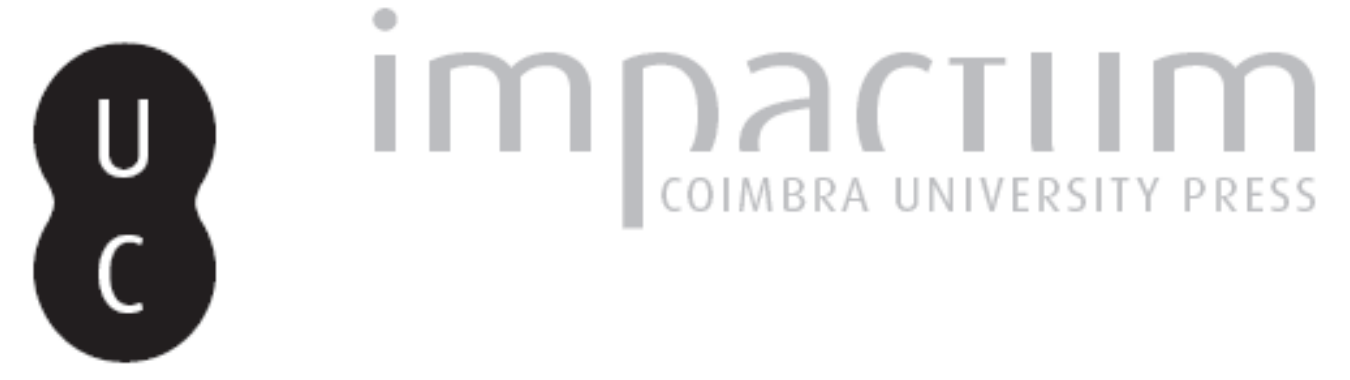

\title{
Propuesta de modificación del baremo español de tráfico para la valoración del daño corporal en el aparato estomatognático
}

Autor(es): $\quad$ López-Nicolás, M.; Oliver, JA.; Muñoz, E.; Luna, A.

Publicado por: Imprensa da Universidade de Coimbra

URL persistente:

URI:http://hdl.handle.net/10316.2/33260

DOI:

DOI:http://dx.doi.org/10.14195/1647-8630_23_3

Accessed : $\quad$ 26-Apr-2023 14:44:59

A navegação consulta e descarregamento dos títulos inseridos nas Bibliotecas Digitais UC Digitalis, UC Pombalina e UC Impactum, pressupõem a aceitação plena e sem reservas dos Termos e Condições de Uso destas Bibliotecas Digitais, disponíveis em https://digitalis.uc.pt/pt-pt/termos.

Conforme exposto nos referidos Termos e Condições de Uso, o descarregamento de títulos de acesso restrito requer uma licença válida de autorização devendo o utilizador aceder ao(s) documento(s) a partir de um endereço de IP da instituição detentora da supramencionada licença.

Ao utilizador é apenas permitido o descarregamento para uso pessoal, pelo que o emprego do(s) título(s) descarregado(s) para outro fim, designadamente comercial, carece de autorização do respetivo autor ou editor da obra.

Na medida em que todas as obras da UC Digitalis se encontram protegidas pelo Código do Direito de Autor e Direitos Conexos e demais legislação aplicável, toda a cópia, parcial ou total, deste documento, nos casos em que é legalmente admitida, deverá conter ou fazer-se acompanhar por este aviso.

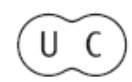


REVISTA PORTUGUESA

$\stackrel{\infty}{\cdots}$

$\stackrel{2}{i}$

$\dot{\bar{x}}$

$\bar{x}_{\bar{x}}$

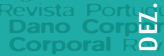

Oeno dorporal Revista Portuguesta do Dano Corporal Revista Portuguens do Dano Corporal

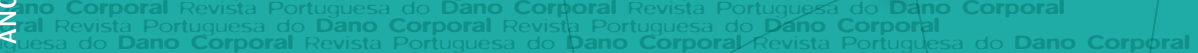

Rvista Portuguesa do Dano Corporal Revista Portuguesa do Dano Corporal Revista Portuguesa do pang Coporal

NPortuguesa do Dano Corporal Revista Portuguesd do Dano Corporal

Silo

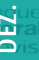

\section{APADAC}

ASSOCIAÇÃO PORTUGUESA

DE AVALIAÇÃO

DO DANO CORPORAL

INSTITUTO NACIONAL

DE MEDICINA LEGAL, I.P.

DELEGAÇÃO DO CENTRO 


\title{
Propuesta de modificación del baremo español de tráfico para la valoración del daño corporal en el aparato estomatognático
}

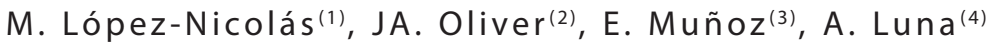

\section{INTRODUCCIÓN}

En España, la valoración del daño corporal en el sistema bucal se encuentra sujeta a limitaciones y dificultades que provienen de la deficiente enumeración y valoración de las secuelas en los actuales baremos vigentes.

Actualmente los baremos más ampliamente utilizados en España son el incluido en la Ley 30/1995, de 8 noviembre. Esta Ley se encarga de la ordenación y supervisión de los seguros privados. Con posterioridad esta Ley fue modificada con la Ley 34/2003 de 4 de noviembre, para adaptarnos a la Normativa Comunitaria de La Legislación de Seguros Privados. El texto refundido de esta Ley fue aprobado pro el Real Decreto 8/2004 de 29 de octubre. Posteriormente con la Ley 21/ 2007 de 11 de julio se modificó el texto refundido de la Ley aprobada por R.D de 8/2004 y R.D 6/2004 de 29 de octubre. Pero en nuestro medio, el baremo utilizado es el recogido por la Ley 34/2003 de 4 de noviembre, que tiene carácter vinculante en las lesiones de tráfico. Además también suele utilizarse el baremo de la Asociación Médica Americana (AMA) y la guía Melennec (Melennec, 1991). Estos baremos son los que nos proporcionan el marco normalizado para analizar y evaluar cualquier aparato o sistema del organismo.

\footnotetext{
Profesor Titular, Facultad de Medicina/Odontología, Universidad de Murcia, España

2 Doctor por la Universidad de Murcia; Colaborador Docente, Universidad de Murcia; Ortodoncista practica exclusiva; España

3 Profesor Asociado de Odontología Forense, Universidad de Murcia; España

4 Catedrático de Medicina Legal y Toxicología, Universidad de Murcia; España
} 
Estos baremos presentan grandes deficiencias y limitaciones (Alonso, 1990; Margeat, 1998)) que nos alejan de la finalidad última de los mismos, que no es otra que el resarcimiento integro de los daños (Hinojal, 1996; Hinojal 2008). La valoración del daño corporal y la reparación a la que tenía derecho toda persona lesionada, sobre todo indemnizaciones de tipo económico, es un tema que siempre ha preocupado al Derecho, y que continuamente está en revisión (Borobia, 1989; Borobia 1996; Borobia, 1996; Borobia 2006).

El presente estudio trata de analizar la situación actual y elaborar una propuesta de modificación del actual baremo de tráfico en el ámbito bucodental, con especial atención a las secuelas de la ATM.

\section{OBJETIVOS Y MATERIAL Y METODOS}

Debido a la ausencia de criterios operativos lo suficientemente amplios para poder evaluar las lesiones odontoestomatológicas atendiendo a sus repercusiones funcionales. Pretendemos establecer criterios que permitan la valoración ajustada a las posibilidades técnicas actuales.

2.1. Esto supone, la elaboración de los siguientes objetivos:

1-Análisis crítico de las tablas y baremos existentes en la actualidad

2-Revisión bibliográfica de los criterios de valoración odontoestomatológica existentes en la actualidad

3-Elaboración de una metodología de valoración del daño adaptada a la clínica odontoestomatológica.

4-Propuesta de unos criterios de valoración del daño anatomofuncional de la articulación temporomandibular.

5-Ver las repercusiones de cada lesión y valorar exhaustivamente su repercusión como función de grupo y aisladamente

6- Revisión de la ley baremo de tráfico $30 / 95$ con todas las modificaciones hasta el presente año 2012 al igual que las guías AMA y Melennec .

2.2. Para la realización de nuestro trabajo, se ha realizado una revisión de las diferentes tablas, estudiando los parámetros que en ellas se utilizan, desde un punto de vista anátomo-funcional. Hemos revisado los distintos componentes de la cinética mandibular (Dawson, 1991), así como la metodología utilizada en el peritaje de la articulación temporomandibular, y los distintos elementos empleados para la evaluación funcional. Destacamos entre ellos, la fuerza máxima de mordedura como un indicador funcional de los trastornos del sistema estomatognático (Duygu, 2010; Bakke, 2006; Calderon, 2006; Pereira, 2007) al igual que los medios diagnósticos de exploración clínica y 
pruebas complementarias, habitualmente utilizados. Algunas de estas pruebas adquieren una especial relevancia a la hora de evaluar el estado de la ATM, como sucede con la electromiografía (Felicio, 2007), el estudio de la oclusión con T-Scan que nos permite valorar el estado de los músculos masticatorios (Freire, 2011), y su implicación funcional, etc. Pero como no siempre es posible utilizar este tipo de pruebas complementarias, nos vamos a centrar, fundamentalmente, en la exploración clínica de esta compleja articulación.

Se han revisado todos aquellos aspectos medico legales, que desde un punto de vista de la articulación temporomandibular, van a ser de utilidad en la valoración del daño corporal. Desde el punto de vista funcional, se clasificaron las lesiones más frecuentes, contemplando en que medida afectaban a las funciones del aparato estomatognático, especialmente la masticación y deglución, en estrecha relación con los desordenes temporomandibulares (Al-Azzawi, 2008), así como sus consecuencias sobre la fonación.

Para el diagnóstico de las alteraciones témporo-mandibulares es fundamental la exploración de músculos y articulación y se requiere un entrenamiento adecuado (Frincton, 1986). Se precisa una adecuada valoración de los movimientos mandibulares, incluyendo la palpación de músculos y articulación (Dworkin, 1992).

\section{PROPUESTA DE CRITERIOS DE VALORACIÓN}

Un baremo siempre estará incompleto. La totalidad de las secuelas que un ser humano puede padecer no es posible recogerlas. La valoración, cuantificación e indemnización de secuelas debe hacerse al amparo de criterios médicos que se utilizan habitualmente en la valoración clínica. (Hernandez-Cueto, 2004). A consecuencia de un traumatismo pueden desencadenarse Trastornos Temporo-Mandibulares con una gran repercusión funcional por alteración en la oclusión habitual (Ohrbach, 2010), que no solo va a repercutir en la masticación, sino también puede desencadenar dolor y gran limitación de los movimientos mandibulares (Carroll, 2007). Estas alteraciones, mas frecuentes en la mujer, pueden aparecer en el momento del accidente o desarrollarse con posterioridad, estando estrechamente vinculadas al traumatismo previo recibido (Epstein, 2010).

A continuación vamos a analizar el sistema del Baremo de Tráfico Ley 30/1995 de 8 de noviembre y la actualización Ley 21/2007 de 11de julio, centrándonos en los diferentes aspectos de la Tabla 2, cara (Sistema Oseoarticular).

Alteración traumática de la oclusión dental por lesión inoperable (consolidación viciosa, pseudoartrosis del maxilar inferior y/o superior, pérdida de sustancias, etc.). 
Con contacto dental:

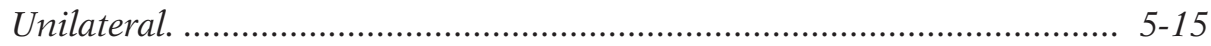

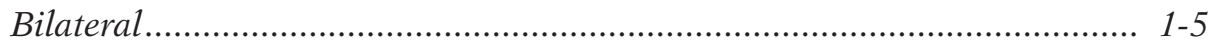

Sin contacto dental....................................................................... 15-30

Deterioro estructural de maxilar superior y/o inferior

(sin posibilidad de reparación). Valorar según repercusión

funcional sobre la masticación ............................................................ 40-75

Pérdida de sustancia (paladar duro y blando):

Sin comunicación con cavidad nasa................................................ 20-25

Con comunicación con cavidad nasal (inoperable)............................. 25-35

Limitación de la apertura de la articulación témporo-mandibular

(de 0 a $45 \mathrm{~mm}$ ) según su repercusión .................................................. 1-30

Luxación recidivante de la articulación témporo-mandibular:

Luxación entre los 20-45 mm de apertura ........................................... 5-10

Luxación entre los 0-20 mm de apertura ........................................ 10-25

Subluxación recidivante de la articulación témporo-mandibular............ 1-5

Material de osteosíntesis....................................................................... 1-8

\section{Boca}

Dientes (pérdida completa traumática):

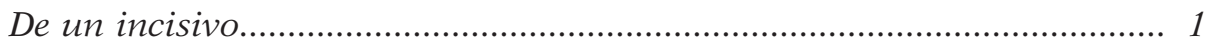

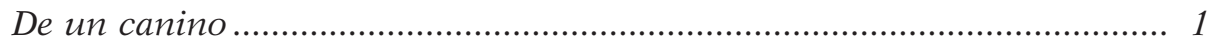

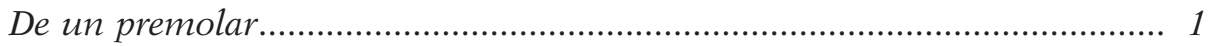

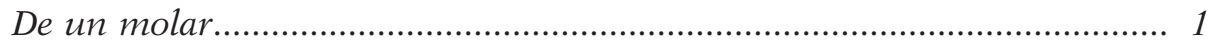

Lengua:

Trastornos cicatriciales (cicatrices retráctiles de la lengua que originan alteraciones funcionales (tras reparación quirúrgica........... 1 1-5

Amputación:

Parcial:

Menos del 50 por ciento

Más del 50 por ciento .............................................................. 20-45

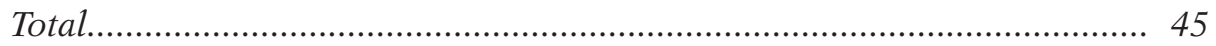

Alteración parcial del gusto ................................................................ 5-12 
A primeros de cada año se actualizan las cuantías indemnizatorias para la valoración de los daños y perjuicios causados a las personas en accidentes de circulación. Con la resolución de 24 de enero de 2012, de la Dirección General de Seguros y Fondos de Pensiones, se publican las cuantías de las indemnizaciones por muerte, lesiones permanentes e incapacidad temporal que resultarán de aplicar durante 2012 el sistema para valoración de los daños y perjuicios causados. (BOE 06-02-2012).

La amplitud de movimiento de la mandíbula se mide desde el borde del incisivo superior al inferior durante la apertura bucal con una regla milimetrada. La apertura de mandíbula mínima normal, es para algunos autores, aproximadamente de $40 \mathrm{~mm}$. El movimiento lateral debería ser 7-10 mm a ambos lados, derecha e izquierda. El movimiento de protrusión normal está entre 6 y 9 mm. (Aragón, 2005).

3.1 La limitación de la apertura de la cavidad bucal presenta una serie de limitaciones según su amplitud:

- Limitación apertura de 0 - 45 mm

- Cuando se presenta una limitación de apertura de 45 a 35 pueden presentarse alteraciones pero que afectaran poco a la fonación, deglución y masticación.

- De 35 a $25 \mathrm{~mm}$ de apertura hay limitaciones pero con amplias posibilidades funcionales de masticación, deglución y fonación. Los cóndilos pueden ser desplazados, produciéndose el movimiento de protrusión.

- De16 a 18 mm de apertura es la altura necesaria para poder comenzar el movimiento de la masticación donde se conjuga un movimiento de lateralidad de 5 a $6 \mathrm{~mm}$ en esta primera fase se atrapa el alimento al aproximar los dientes $3 \mathrm{~mm}$ y el alimento se machaca entre las cúspides de los dientes y donde la mandíbula se desplaza de 3 a $4 \mathrm{~mm}$ en un movimiento lateral.

- De 15 a 0 mm de apertura las limitaciones para la lateralidad sólo dan margen de 5 a $6 \mathrm{~mm}$ y luego en la formación del bolo alimenticio de 3 a 4 $\mathrm{mm}$ con lo cual la alimentación se restringe a una dieta blanda o líquida.

En cuanto a la fonación se restringe sonidos agudos al no poder descender la mandíbula por la apertura mandibular.

La ingesta de líquidos está comprometida con menos de $5 \mathrm{~mm}$.

Para la valoración se puede aplicar una recta de ajuste entre la alteración funcional y la puntuación asignada, como se recoge en la figura siguiente, en la que hemos representado una función lineal (Tabla 1)

Aplicar una función lineal no parece razonable ya que las repercusiones son más intensas en el último tercio de apertura y requiere una matización en función de la limitación global resultante, por esto consideramos que sería más real ajustar una función exponencial a partir de una limitación de 
$18 \mathrm{~mm}$ como recogemos en la figura siguiente (Tabla 2). Discrepando de las puntuaciones sugeridas por otros autores (Perea, 2007).

Tabla 1. Crecimiento uniforme según Ley Baremo 30/1995 (actualización 21/2007)

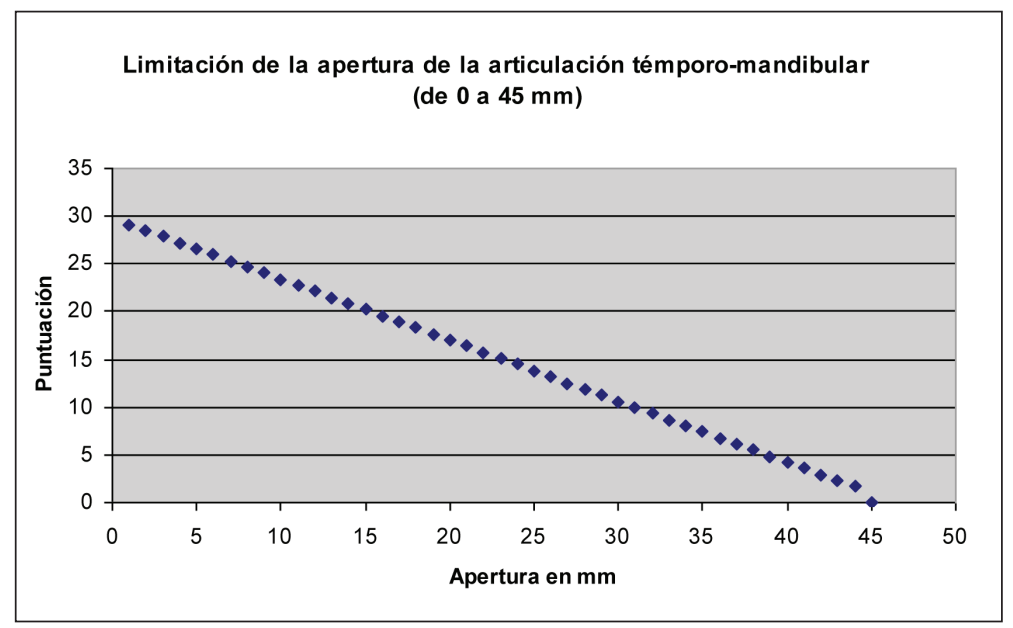

Tabla 2. Crecimiento exponencial a partir de $18 \mathrm{~mm}$, según nuestra propuesta, donde queda afecta las funciones masticatorias se ha incrementado 1/3 de 18mm y $1 / 3$ de 12 - $6 \mathrm{~mm}$ para que queden compensados las deficiencias que padece nuestro sistema de baremación Ley Baremo 30/1995 (actualización 21/2007)

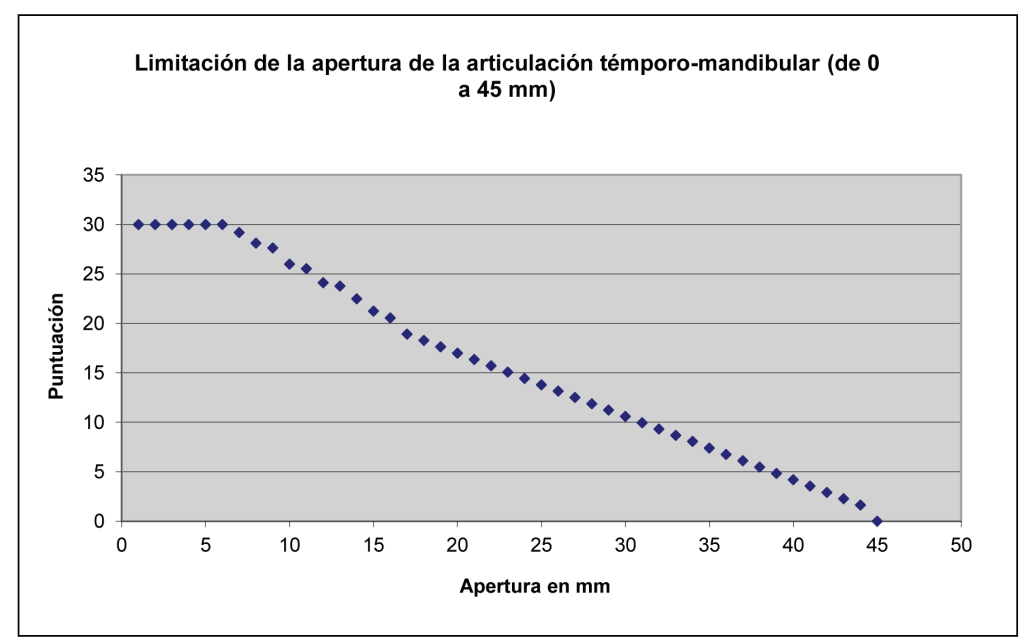


3.2 Alteración traumática de la oclusión dental por una lesión inoperable con contacto dental.

El Baremo de Tráfico no tiene en cuenta el tipo de traumatismo de que se trata si es una pseudoartrosis extraarticular, o bien una anquilosis verdadera de tipo intrarticular (en sus diversas clasificaciones ósea, fibrosa, fibroósea y cartilaginosa).

La característica común de las anquilosis, o cualquier tipo de traumatismo que produzca una hipomovilidad articular es la falta de movilidad de la articulación. Dependiendo de la dificultad de la fonación y del paso de líquidos en el baremo tiene una puntuación errónea ya que las condiciones de afectación a todos lo niveles, fonación, masticación deglución requieren una mayor puntuación. En el baremo solo se evalúa si hay contacto dental siendo unilateral o bilateral dependiendo del número de piezas o de la superficie dentaria afectada. En el caso de la ausencia de contacto dental la ingesta alimenticia es a base de dieta líquida y con otros problemas añadidos.

Desde nuestro punto de vista este resulta manifiestamente injusto ya que seria necesario incluir la superficie real de contacto y en función de la misma aplicar un coeficiente de corrección que refleje la precisión funcional resultante de la falta de contacto.

La perdida de uno o mas dientes por causa traumática o deterioro de los maxilares no tratada protésicamente tiene múltiples consecuencias sobre la zona concreta de daño y sobre la función global del aparato estomatognático. Entre las alteraciones que puede producirse están:

$\boldsymbol{A}$ - Las alteraciones de la función masticatoria: Con la perdida de dientes, menor capacidad y eficiencia masticatoria. La capacidad masticatoria se mide según el tamaño de los fragmentos alimenticios después de un numero determinado de ciclos. La eficiencia masticatoria se basa en el numero de ciclos masticatorios necesarios para fragmentar los alimentos de un tamaño determinado. Esto se traduce en menor número de ciclos masticatorios, ulceras en la mucosa gástrica.

Las alteraciones de la función masticatoria depende no solo del numero de dientes que faltan, sino de la posición que ocupan y el tipo de diente. Hay que recordar que las unidades masticatorias no son dientes aislados, sino los trígonos dentarios formados por un diente y los dos antagonistas con los que articula. Así la pérdida de dos dientes puede afectar a un numero distinto de trígonos según la localización de las piezas.

$\boldsymbol{B}$ - Movimientos dentarios como inclinaciones, extrusiones, rotaciones y formación de diastemas. 
$\boldsymbol{C}$ - Aumento de la carga oclusal sobre los dientes remanentes: Al faltar piezas dentales, aunque la fuerza dental disminuye, todas las demás soportan un sobrepeso, Esto provoca un aumento de la carga en el ligamento periodontal que puede dañar.

$\boldsymbol{D}$ - Patología en la Articulación Temporo-Mandibular: Si se pierden los sectores posteriores total o parcialmente, parte de la carga oclusal se desplaza a la ATM y puede darse un síndrome de dolor disfunción de la ATM.

$\boldsymbol{E}$ - Alteraciones fonéticas. Aparece cuando el numero de dientes ausentes es elevado. Los problemas mayores se encuentran en la perdida del grupo de dientes anteriores, ya que son necesarios para la correcta pronunciación de consonantes interdentarias, labiodentales etc.

$\boldsymbol{F}$ - Alteraciones estéticas.

Como podemos observar en las figuras siguientes (Tablas 3 y 4) en el baremo actual no existe una correspondencia real entre la repercusión funcional y la valoración que se refleja en las tablas correspondientes según lo enunciado anteriormente. Por lo tanto consideramos que se evalúe por sectores; anterior medio y posterior y cada sector tendrá un valor dependiendo de la superficie de contacto. Nosotros aumentaríamos la puntuación debido a las repercusiones funcionales analizadas en contacto unilateral otorgaríamos de 5 a 25 puntos y bilateral de 1 a 10 puntos, según zona de contacto dental. (Tabla 5 y 6).

Tabla 3. Según Ley Baremo 30/1995 (actualización 21/2007). Alteración traumática de la oclusión por lesión inoperable con contacto dental unilateral

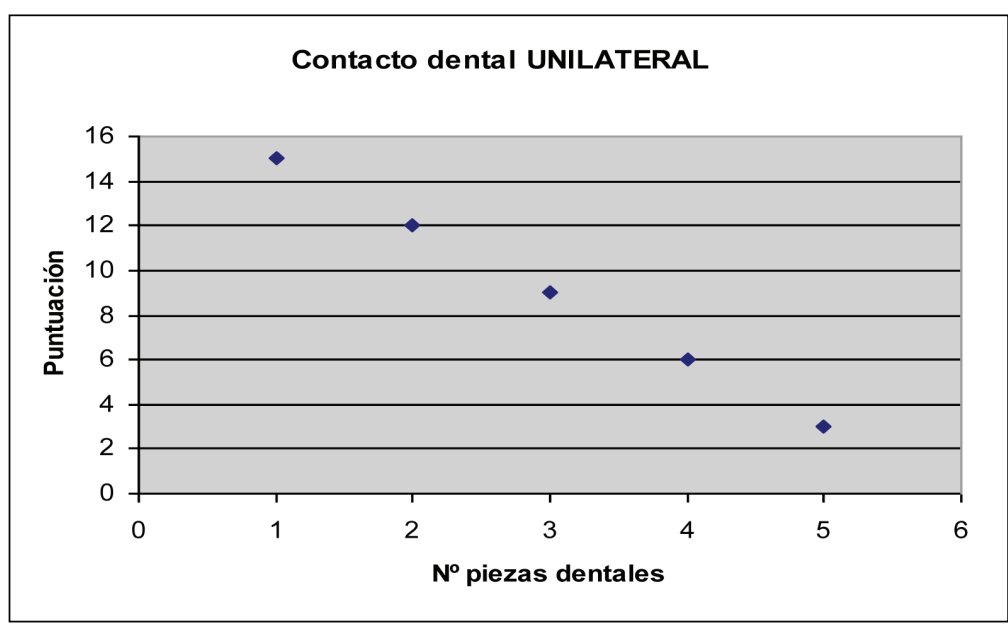


Tabla 4. Según Ley Baremo 30/1995 (actualización 21/2007). Alteración traumática de la oclusión por lesión inoperable con contacto dental bilateral

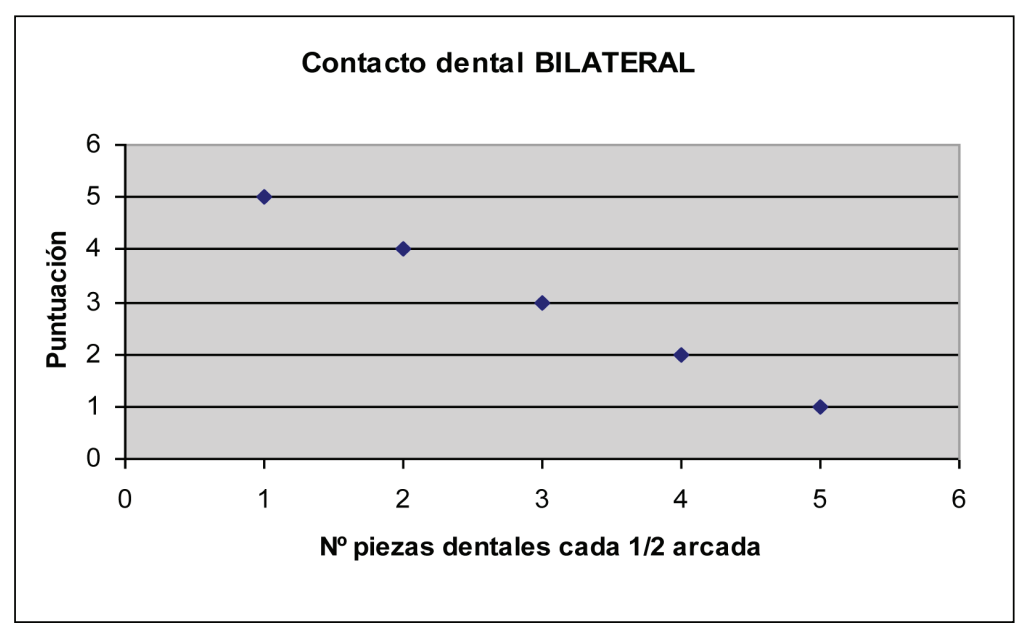

Tabla 5. Según nuestra propuesta donde hemos realizado un aumento de los valores que nos parecen más justificados

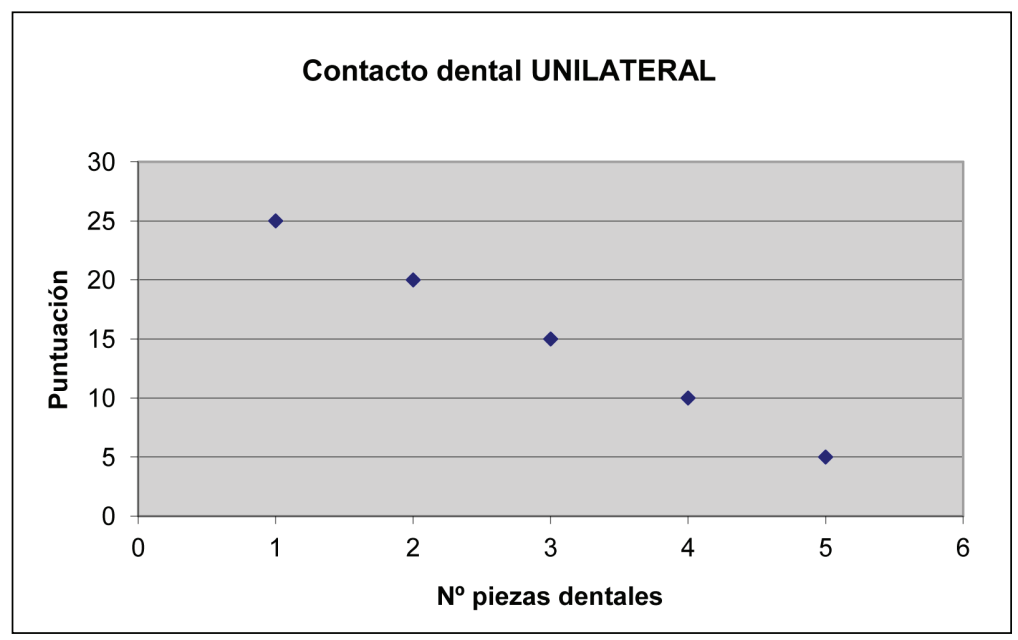


Tabla 6. Según nuestra propuesta incluyendo el aumento de las puntuaciones más justificadas los valores

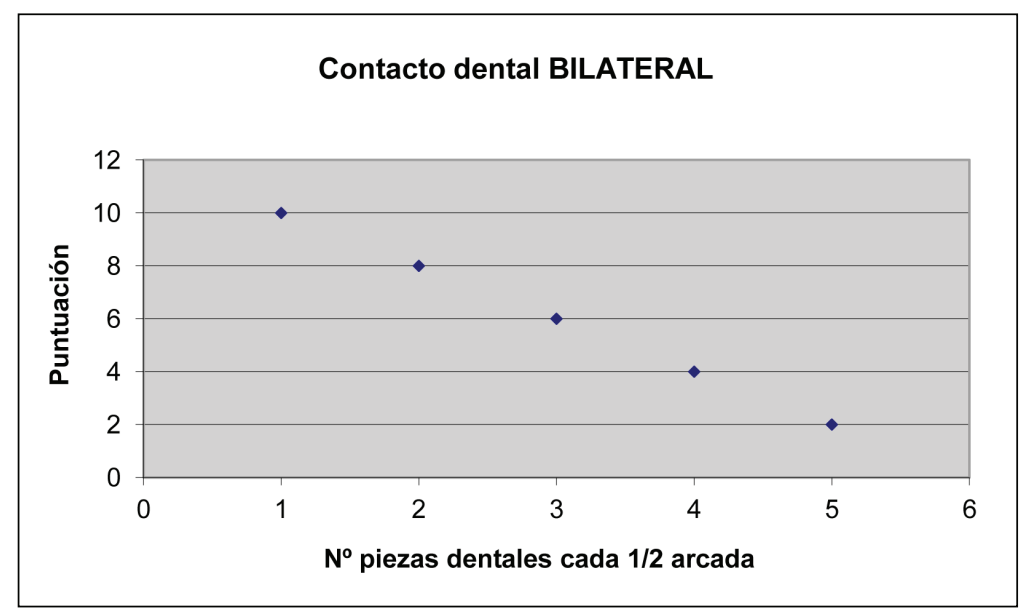

3.3 Deterioro estructural del maxilar superior y /o inferior (Sin posibilidad de reparación). Valor según repercusión funcional sobre la masticación.

Las fracturas mandibulares las podemos clasificar por su región anatómica sinfisiaria (media o parasinfisiaria) que comprende la región delimitada por distal de los caninos, cuerpo desde distal de la sínfisis hasta una línea coincidente con el margen alveolar del músculo masetero; ángulo; región triangular delimitada por el margen anterior del músculo masetero hasta su inserción posterosuperior, rama ascendente delimitada por el margen superior del ángulo hasta la escotadura sigmoidea; cóndilo y apófisis coronoides y región alveolar.

En ningún baremo se analiza la zona afectada en el caso de pérdida Creemos que la puntuación máxima dada en el caso de pérdida importante según la repercusión sobre la masticación no creemos que pueda suplir tal perdida ya que no hay posibilidad de reparación , nosotros atribuiríamos de un 50 a un $100 \%$.

\subsection{Luxación recidivante de la ATM.}

Los principales síntomas y disfunciones de los trastornos de la ATM se asocian a una alteración del complejo cóndilo-disco. Los pacientes refieren a menudo una artralgia, pero la disfunción es la manifestación más frecuente. Los síntomas de disfunción se asocian al movimiento condileo y se describen como sensaciones de "clic" o de atrapamiento de la articulación. Suelen ser constantes y repetitivos y a veces progresivos. Las alteraciones del complejo cóndilo-disco tienen su origen en un fallo de la función de rotación normal 
del disco sobre el cóndilo. El factor etiológico más frecuente asociado son los traumatismos. Nosotros proponemos un aumento en la puntuación de 0-20 $\mathrm{mm}$ de apertura.

3.5 Subluxación recidivante de ATM.

Dependiendo el grado de hipermovilidad que presente el paciente y las veces que se produce la subluxación. Se caracteriza por la presencia de un chasquido durante la apertura y cierre. Cuando hay dolor se trata de una molestia preauricular que se suele irradiar a oído, mismo lado de la cara y se exacerba con la función. En nuestro análisis y estudio realizado es lógica la puntuación que recibe el sistema de baremación de la Ley 30/1995 (actualización 21/2007).

3.6 Movimientos de lateralidad.

En nuestro sistema de baremo de la Ley de Tráfico 30/1995 (Actualización 21/2007) no se contemplan los movimientos de lateralidad, siendo de fundamental importancia en las funciones de masticación, fonación y deglución. Nosotros otorgamos una horquilla de puntuación comprendida entre 1 a 10 puntos.

3.7 Movimientos de protrusión.

Los movimientos de protrusión en el sistema masticatorio se dan cuando la mandíbula se desplaza de atrás adelante. Los dientes, los cóndilos y las ramas se desplazan en una misma dirección. La traslación se realiza dentro de la cavidad superior de la articulación entre el complejo disco-cóndilo y la fosa articular. Durante la mayoría de los movimientos normales de la mandíbula, se da una rotación y una traslación.

En la protrusión la distancia mínima para que se realice, es con una distancia interincisiva a partir de 20 a $25 \mathrm{~mm}$. Aproximadamente (Barton, 1996). En nuestra propuesta incluimos un ligero incremento en la horquilla de valores a partir de $20 \mathrm{~mm}$.

El cálculo o la aproximación al porcentaje de pérdida masticatoria en relación a la articulación temporomandibular lesionada resulta menos clara, la función es mucho más difícil de cuantificar según baremo; Mientras que una anquilosis ósea bilateral de la mandíbula sin apertura bucal representa un $100 \%$ de incapacidad, una anquilosis unilateral representa el 50\% de perdida de función.

El baremo tendría que concebir un cálculo basado en la pérdida de la apertura bucal. En el baremo vigente, no se contemplan los movimientos de lateralidad y de protrusión, retrusión. 
En el análisis de los gráficos del sistema oseoarticular del baremo de tráfico 21/2007 vemos un crecimiento rectilíneo uniforme, pensamos que en ciertas lesiones, a partir de ciertos $\mathrm{mm}$. de apertura queda involucrados otros problemas funcionales de mayor entidad, que no son contemplados como son los movimientos de lateralidad retrusión, protusión que son necesarios para las funciones fisiológicas de masticación, deglución, fonación.

Según nuestra propuesta en la masticación queda muy limitada a partir de $25 \mathrm{~mm}$. a partir de $18 \mathrm{~mm}$ ya quedan patentes cambios en la masticacion, ya que son los mm. críticos para que se dé un ciclo en la masticación. En nuestra propuesta se incrementaría $1 / 3$ de $18 \mathrm{~mm}$ a 12 y de $12-6 \mathrm{~mm}$ se aumentaría $1 / 2$ y de 6 a $0 \mathrm{~mm}$ se incrementara $1 / 2$ para que se produzca un crecimiento exponencial y de esta forma se solventen las deficiencias que hemos podido analizar en nuestro sistema de baremación.

Se puede observar al comparar las graficas realizadas según el baremo actual de tráfico y según nuestra propuesta, como el crecimiento de la puntuación es mayor a partir de los mm críticos para que puedan darse las funciones masticatorias.

\section{DISCUSIÓN}

Debido a la ausencia de criterios sistematizados para una adecuada valoración de la articulación temporomandibular hemos decido realizar una propuesta de valoración de las lesiones situadas en esta articulación. Antes de proceder a en la exposición de esta propuesta hemos considerado oportuno sobre una serie de casos clínicos evaluar las insuficiencias de los baremos existentes y aplicar los criterios que proponemos para poner de manifiesto la realidad del problema y la eficiencia práctica de los criterios propuestos.

Somos conscientes de la insuficiencia de los casos clínicos que presentamos a continuación para reflejar en toda su amplitud la realidad clínica posible, sin embargo consideramos que si bien no refleja la totalidad si nos permite asomarnos a aquellas realidades más frecuentes en la valoración del daño corporal.

\section{CASO CLÍNICO No 1}

Paciente varón, 28 años de edad, profesión agente inmobiliario. Acude a consulta remitido por su otorrino con diagnóstico presuntivo de anquilosis de atm referida desde hace un año, la anamnesis pone en evidencia un trauma por accidente por caída en motocicleta, (trauma en mentón con una limitación 
de movimientos mandibulares), a partir del traumatismo el paciente refiere una pérdida progresiva de la apertura bucal sin otra clínica asociada,. Desde hace una semana comienza con un dolor intenso que se refleja en el oído derecho y que se atenúa con analgésicos ibuprofeno $600 \mathrm{mg} / 12 \mathrm{~h}$.

"A la exploración presenta una apertura bucal de $10 \mathrm{~mm}$ sin deflexión del trayecto en la línea media, con gran limitación de los movimientos de lateralidad (3 mm de limitación).Durante la exploración clínica, se aprecia dolor a la palpación en la articulación derecha siendo asintomática la izquierda, a la palpación muscular se evidencia dolor a nivel del masetero, pterigoideo interno y externo del lado derecho.

Al examen radiológico ( $\mathrm{RX}$ panorámica y transcraneal), no se observa un desplazamiento condilar significativo desde la posición de reposo a la posición de apertura, no hay diferencia rx entre la posición de protrusión y laterotrusión. El examen radiológico revela una línea de fractura subcondilar derecha preexistente sin desplazamiento.

En el momento actual la Clínica se manifiesta con una limitación de la apertura bucal, maloclusion dentaria y mordida abierta anterior (en casos bilaterales) y dolor con una apertura limitada de $10 \mathrm{~mm}$ queda comprometida los movimientos para realizar la masticación, al igual que la lateralidad 3mm.

\section{BAREMO ACTUAL 21/2007}

Le correspondería un 78\% de pérdida de función con 30 pt. Si la limitación de la apertura máxima son $45 \mathrm{~mm}$ y el mínimo 0 hay un mínimo de 15 pt y un máximo de 30 pts con una diferencia de 15 si en este caso al ser $10 \mathrm{~mm}$ de apertura sería 1.5 por cada grado, no se evalúan ni las limitaciones de los movimientos de lateralidad ni de protrusión. En nuestra propuesta de baremo ajustaríamos la restricción de los movimientos de lateralidad sumándose a los puntos obtenidos en la limitación de apertura bucal.

Con estas limitaciones el tipo de dieta que puede ingerir seria la consistente en: Frutas sandias, albaricoques, vegetales escasos en fibras y de consistencia suave tomates y en preparaciones diversas cocción etc. carbohidratos: sémolas y pastas de diámetro pequeño lácteos, proteínas animales como pescado, huevos alimentos líquidos como zumos de frutas y verduras etc.

El perjuicio estético, estará afectado en la limitación de la sonrisa y en algunos gestos de la mímica, además de las cicatrices como consecuencia del accidente. 


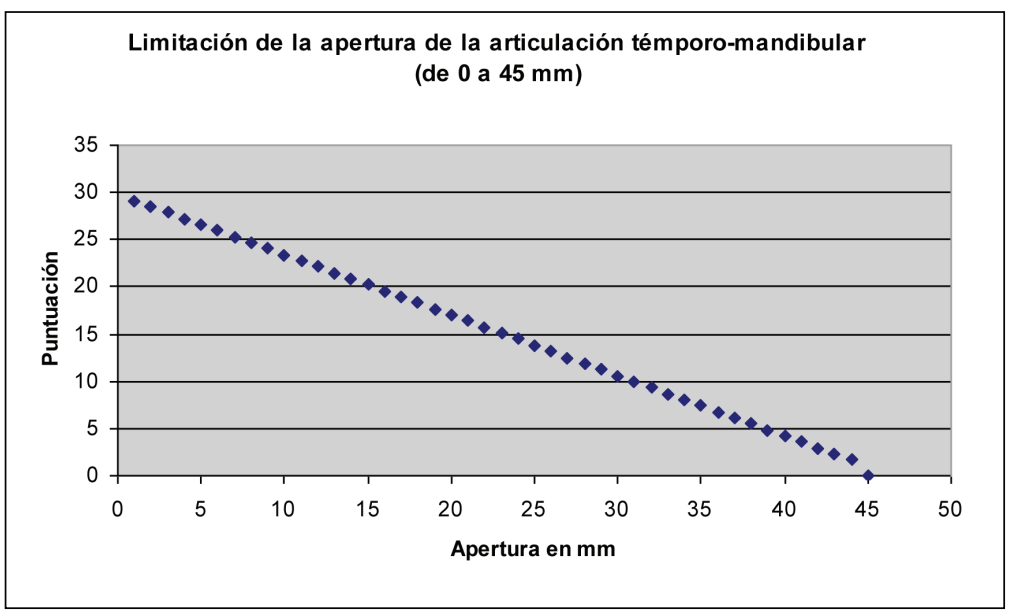

\section{EN NUESTRA PROPUESTA}

Se le otorgaría un valor de 26 pt al aumentar 1/3 el valor de la limitación de la apertura a partir de los $18 \mathrm{~mm}$ necesarios para que se pueda realizar los movimientos normales masticatorios. Aquí incluimos la deficiencia de los movimientos de lateralidad que le daríamos una puntuación de 6 puntos (la horquilla de valoración de la limitación de los movimientos de lateralidad esta entre 10 y 1 pt.), y estos 6 puntos los sumariamos a la puntuación resultante de la hipomovilidad.

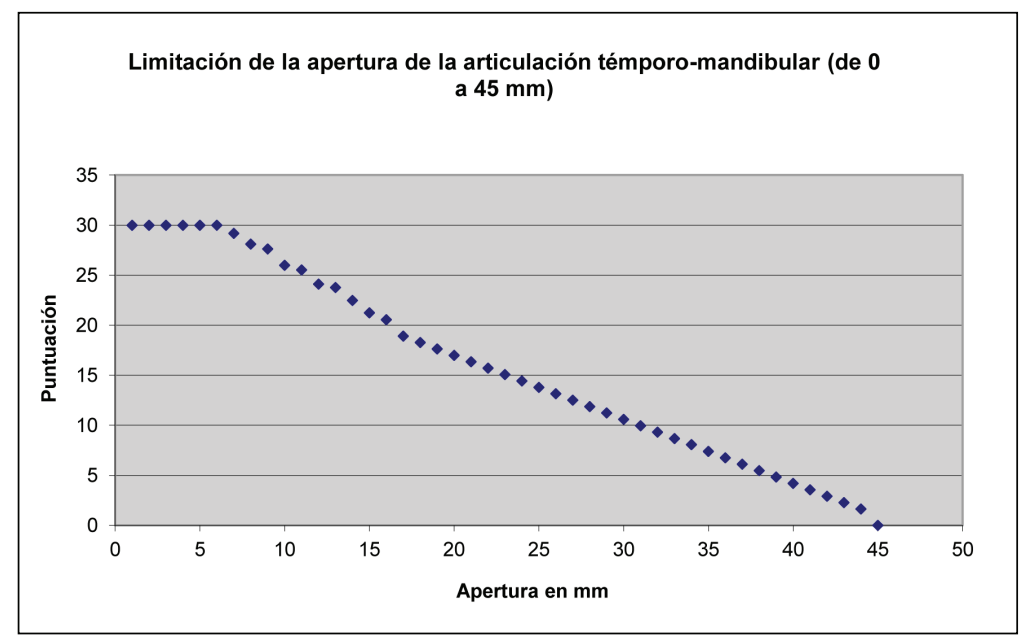

Tabla donde queda de manifiesto nuestra propuesta con un crecimiento exponencial a partir de los $17 \mathrm{~mm}$. 


\section{CASO CLÍNICO No 2}

Paciente de 18 años varón, estudiante de económicas, con dolor intenso en atm izquierda, tras sufrir accidente de tráfico con golpe en cabeza, al día siguiente del accidente noto, que la atm izquierda era dolorosa a la palpación y el dolor iba aumentando de forma progresiva cada día.

En la exploración se observó que a la palpación la articulación presentaba dolor intenso. Presentaba además ruidos articulares y una tumefacción apreciable en el área articular, el dolor era constante con el movimiento mandibular. La apertura de la boca era de $12 \mathrm{~mm}$.

Las radiografías transcraneales revelaron una fractura mandibular en el ángulo mandibular, con limitación en la apertura. En la palpación se observa dolor en el masetero izquierdo y en el temporal derecho. El estado oclusal estaba en los límites correctos sin pérdida dentaria. El diagnóstico era de fractura de ángulo mandibular sin desplazamiento.

El pronóstico es bueno por no tener desplazamiento y la línea de fractura tiene un trayecto anteroinferior sin afectación neurológica. Las repercusiones funcionales, afectan a la función alimenticia masticatoria, también pueden afectar a la función respiratoria y fonatoria, incapacidad transitoria funcional mono articular, con algias permanentes y afectacion en la sonrisa y en la mímica

En cuanto a las dietas y hábitos alimenticios, las posibilidades se reducen al consumo de alimentos semitriturados, blandos, consumo apto de pastas sémolas, purés, pescado, frutas y verduras en purés con bajo nivel de fibras, zumos y lácteos.

\section{BAREMO ACTUAL 21/2007}

Una limitación de la apertura de $12 \mathrm{~mm}$, le correspondería 20 pt en el baremo de tráfico, ya que restringe los movimientos masticatorios, fonéticos, deglución. En este baremo no se tienen en cuenta los movimientos de lateralidad ni los de protusión.

\section{NUESTRA PROPUESTA DE VALORACIÓN}

Sería de 22 pts, porque hemos incrementado 2 puntos al incluir la limitación de los movimientos de lateralidad junto a la limitación de la apertura a partir los $17 \mathrm{~mm}$ necesarios para producirse los movimientos masticatorios. 


\section{CONCLUSIONES}

1- Del estudio realizado resulta evidente la insuficiencia del baremo de tráfico actual para la valoración del daño odontoestomatológico y más concretamente para la valoración de la articulación temporomandibular.

2- El baremo de tráfico actualmente vigente, 21/2007 no contempla los movimientos de lateralidad y protrusión retrusión del sistema oseoarticular de la cara. Nuestra propuesta es la inclusión de los movimientos de lateralidad protusión retrusión que consideramos necesarios, desde un punto de vista anatomo funcional.

3- Los movimientos de lateralidad que no se contemplan en nuestro sistema de baremación le otorgamos una horquilla comprendida entre 1 y 10 puntos.

4- No nos parece razonable asignar la misma puntuación a las distintas piezas dentales teniendo en cuenta su función fisiológica.

5- Consideramos que la puntuación asignada a los caninos debe ser de 1,5 pts a los incisivos y molares 1 pt y a los premolares 0,5 pt.

6- Debe incluirse sistemática una exploración funcional de la masticación en todos los traumatismos faciales incluyendo un análisis cinético de la masticación.

7- Consideramos necesarios la actualización y revisión de los criterios actuales.

\section{REFERENCIAS}

Alonso Santos J. Reparation du prejuice corporel en Espagne (droit común).Rev.Franç dommage corporel 1990; (16): 543-546.

Al-Azzawi TR, Hamdan FB, Ali AK. Neurophysiologic evaluation of the temporomandibular joint and related masticatory muscles in rheumatoid arthritis patients. Neurosciences (Riyadh). 2008; 13(3):253-8.

Aragón MC, Aragón F, Torres LM. Trastornos de la articulación témporo-mandibular. Rev. Soc. Esp. Dolor 2005; 12: 429-435.

Asociación Medica Americana (A. M. A.). Tablas de evaluación del menoscabo permanente B. O. E. de 16y 17 de Marzo (O. M. 6 de Marzo de 1984).

Bakke M. Bite force and occlusion. Semin Orthod 2006;12:120-126. 
Barton J. On the treatment of anchylosis by the formation of artificial joints. Am.Med. Surg.J.1996; (3): 279-292.

Borobia Fernández C Métodos de Valoración del daño Corporal. Madrid: Fundación Universidad Empresa; 1989: 30 -158.

Borobia Fernández C. Valoración de Daños Corporales causados en los accidentes de circulación. Madrid. Fundación Universidad Empresa; 1996.

Borobia Fernández, C. El nuevo Baremo Legal y los defectos científicos (Anexo de la ley 30/95) Problemática en la valoración medica de las secuelas. En rev. De Responsabilidad Civil, Circulación y Seguro. Ed. Inese, Enero 1996 4-8.

Borobia Fernández, C. Valoracion del Daño Corporal, Legislación Metodología y prueba pericial Médica, Elservier Masson 2006.

Calderon Pdos S, Kogawa EM, Lauris JR, Conti PC. The influence of gender and bruxism on the human maximum bite force. J Appl Oral Sci 2006; 14: 448-453.

Carroll, LJ., Ferrari, R., Cassidy, JD., Reduced or painful jaw movement after collisionrelated injuries. The Journal of the American Dental Association 2007; 138 (1): 86-93.

Dawson, P. Evaluación, Diagnostico y tratamiento de los problemas oclusales. Barcelona. Ed. Salvat; 1991.

Duygu K., Arife D., Budent B., Bite Force and Influential Factors on Bite Force Measurements: A literature Review. European Journal of Dentistry 2010; 4: 223-232.

Dworkin SF, LeResche L. Research diagnostic criteria for temporomandibular disorders: review, criteria, examinations and specifications, critique. J Craniomandib Disord 1992; 6: 301-55.

Epstein, JB., Klasser,GD., Kolbinson, DA., Mehta, SA. Orofacial Injuries Due to Trauma Following Motor Vehicle Collisions: Part 2. Temporomandibular Disorders. Can Dent Assoc 2010; 76 a 172.

Felicio CM., Melchior MO., Silva MAMR., Celeghini RMS. Desempenho mastigatório em adultos relacionado com adesordem temporomandibular e com a oclusão. Pró-Fono Revista de Atualização Científica 2007; 19, 2, 151-158.

Freire Matos M, Durst AC, Freire Matos JL, Learreta JA. Electromyographic evaluation of the 'vertical' dimension: the Learreta TMJ decompression test. Cranio. 2011; 29(4):255-60.

Frincton J, Schiffman E. Realiability of a craniomandibular index. J Dent Res 1986; 65: 1359-64.

Hernández-Cueto C. Análisis Médico Legal de la Nueva Tabla de la ley sobre responsabilidad Civil y Seguro en la circulación de vehículos de motor (Ley 34/95, de 4 de noviembre, de modificación y adaptación comunitaria de la legislación sobre seguros privados) 2004;(12):2739.

Hinojal Fonseca, R. Daño corporal, fundamentos y métodos de valoración médica. Madrid. Arcano médica; 1996: 15-43.

Hinojal Fonseca, R. Discurso de Entrada en la Real Academia de Medicina del Principado de Asturias (La valoración del daño a la persona y la Medicina Legal) 2008.

Margeat H. La doctrine de la reparation du damage corporel. Revue Française du Dommage Corporel 1998; (14): 62.

Melennec, Louis. Bareme International des invalidites postraumatiques. Paris: Editorial Masson; 1991. 
Ohrbach R. Disability assessment in temporomandibular disorders and masticatory system rehabilitation. J Oral Rehabil. 2010;37(6):452-80.

Perea Pérez B., Labajo González E., Santiago Sáez A., et al. Propuesta de una metodología de exploración y de valoración de las secuelas de la articulación témporo mandibular (ATM) Mafre Medicina, 2007;18 (1): 18-26.

Pereira LJ, Pastore MG, Bonjardim LR, Castelo PM, Gavião MBD. Molar bite force and its correlation with signs of temporomandibular dysfunction in mixed and permanent dentition. J Oral Rehabil 2007; 34: 759-766.

Resumo: Proposta de alteração da tabela espanhola para avaliação do dano corporal por acidentes de viação na cavidade oral

Em Espanha, a avaliação do dano corporal na cavidade oral está sujeita a limitações e dificuldades provenientes da deficiente descrição e valorização das sequelas nas atuais tabelas em vigor. Neste estudo analisa-se e elabora-se uma proposta de modificação da atual tabela espanhola para avaliação do dano corporal por acidentes de viação no que respeita à cavidade oral, com especial atenção às sequelas da ATM - Articulação Temporomaxilar- (TMD). No estudo efetuado evidencia-se a insuficiência da tabela atual para a avaliação do dano na cavidade oral e mais concretamente para a avaliação da articulação temporomaxilar. A tabela atualmente vigente não contempla os movimentos de lateralidade, protrusão e retrusão do sistema osteoarticular da face. Propomos a inclusão dos movimentos de lateralidade, protrusão e retrusão que consideramos necessários do ponto de vista funcional. Para os movimentos de lateralidade, não contemplados na nossa tabela, propomos uma valorização entre 1 e 10 pontos. Não nos parece razoável atribuir a mesma pontuação às distintas peças dentárias dada a sua diferente função fisiológica. Assim, consideramos que a pontuação a atribuir aos caninos deve ser de 1,5 pontos, aos incisivos e molares de 1 ponto e aos pré-molares de 0,5 pontos.

Palavras-Chave: Dano corporal; tabela para avaliação do dano corporal por acidentes de viação; dano oral; avaliação forense.

Summary: Proposal to amend the Traffic Spanish scale for assessment of the bodily damage in the oral cavity

In Spain the assessment of the corporal damage within the oral system is subject to limitations and difficulties which are caused from the deficient enumeration and assessment of the after-effects in the current and valid scales. The present study deals with the analysis and the elaboration of a proposal to modificate the current traffic scale in the bucco-dental field. It pays special attention to the after-effects of the TMD (Temporomandibular Disorder). The carried out study bears witness to the insuffiency of the scale of the current traffic in order to evaluate the dental-stomatological damage and, specially, in order to evaluate the temporomandibular joint. The traffic scale, which is currently valid, does not consider the protrusion, lateral and retrusion movements of the osteoarticular system of the face. Our proposal is to include the protrusion, lateral and retrusion movements that we consider 
as necessary from a functional viewpoint. We give a wye between the points between 1 and 10 . We do not find reasonable to assign the same valoration to different dental pieces bearing its different physiological function in mind. Therefore, we consider that the punctuation assigned to canines should be 1.5 punts for incisors; 1 point for molars and 0,5 for premolars.

Key-Words: Corporal damage; traffic scale; oral damage; forensic assessment.

\section{Pedido de separatas:}

MANUEL LÓPEZ NICOLÁS

malopez@um.es 